\title{
Research and Application of Machine Vision Technology in Workpiece Detection and Recognition
}

\author{
Xiangwei Chen ${ }^{1}$ and Tiantian Zhang ${ }^{2, *}$ \\ ${ }^{1}$ School of mechanical engineering, Northeast Electric Power University, Jilin 132012, China \\ ${ }^{2}$ Mechanical engineering and automation, Northeast Electric Power University, Jilin 132012, China \\ "Corresponding author
}

\begin{abstract}
This paper adopt image recognition to realize the defect detection of small probability defect parts in cluster mode. In this mode, parts with small probability of defects were identified one by one to be recognized. Different lighting conditions were tried to determine what kind of lighting should be used for auxiliary lighting. By means of steps such as gray scale stretching and threshold segmentation, the collected image could be used as binary graph suitable for defect detection after preprocessing. The characteristics of the bolt itself were used as the identification condition to match the measured item with the template. Then the difference information between the matching result and the template was calculated by difference shadow method. And according to this information, whether the product to be tested is qualified is determined.
\end{abstract}

Keywords-defect defecting; cluster mode; image identification

\section{INTRODUCTION}

Due to a variety of factors during the production process such as technology, precision, accidents, etc., there will be a certain proportion of defective products in the finished product that do not meet the requirements of the factory, and the presence of these products reduces the entire qualified rate. And the insufficient qualified rate will course influence in the subsequent processing or use to a certain extent. Therefore, the elimination of the defective parts produced in the production line has become an important means to the improvement of overall qualification rate of the products. Defect detection of the products reduces the yield of the products to a certain extent, and in some cases it can recycle the defective parts directly before they leave the factory, which reduces the waste of resources.

There are many methods for defect inspection at present, and different methods are used for different situations of defect. For example, ultrasonic waves are often used as a detecting means ${ }^{[1]}$ on the detection of crack gap of the internal structure of the product, and for surface defects, the most common way to detect the defect is image recognition ${ }^{[2]}$.

Defect detection using image recognition overcomes the uncertainty of manual detection, and does not cause omissions due to fatigue ${ }^{[3]}$ in a large number of repetitive tasks, which greatly reduces the manpower, and its non-contact detection method also make it possible to avoid the damage caused by touching in the detection of some precision parts.
At present, there are many ways of using image recognition in defect detection. Depending on the development of computer technology, many algorithms of image recognition that can adapt to many different situations has been produced. Most commonly used algorithms are difference method, convolutional neural network and BP neural network ${ }^{[4]}$.

The parts of cluster mode, due to their high output and other characteristics, a small number of defective parts appear during production. It is a very important step in the process to identify and select these defective parts. In this project, bolts are intended to be selected as detection objects. The surface defects of the bolt during its processing are divided into several types according to its origin and position. Among them, the common bolt body defects are burrs, split angles, insufficient teeth, tails to the tail, masts, Rough bottom of the teeth, etc. ${ }^{[5]}$.In this paper, the tail teeth are not rubbing to the tail tip and no teeth, so as to carry out defect detection by programming.

\section{LIGHTING SELECTION}

In the process of defect detection, suitable lighting is one of the most important conditions to ensure the smooth progress of inspection. Suitable illumination can make the object to be measured and the background have enough difference of grayscale after the image is grayed, and make it possible to be clearly and simply distinguished from the background in the threshold segmentation $\operatorname{step}^{[6]}$, while the illumination of the highly reflective complex surface must be kept at a relatively dark level, such as bolts, internal threads, etc. Excessive light and direct light will produce excessively specular reflection on the surface. This specular reflection will increase the brightness of the object tested in the picture, which leads to the relationship between the object been tested and the background in the picture. When the contrast between the two is reduced, the decrease in the gray level difference will affect the accuracy of the threshold segmentation, further affecting the degree of completion of the contour recognition. Therefore, the selection of appropriate illumination is one of the most important factors to consider in image recognition defect detection ${ }^{[7]}$.

Natural light is the most securable and most common way of illumination. Image recognition under natural light can keep expenses low, but natural light also has its unfavorable factors. First, it is greatly affected by time and light intensity. The incident angle and duration are uncontrollable. Figure 1-a shows the bolt image taken under natural light. It can be seen 
from the figure that the natural light is too low for the image to be clear enough, which can not completely satisfy the separation of the object to the background (Fig. 1-b).
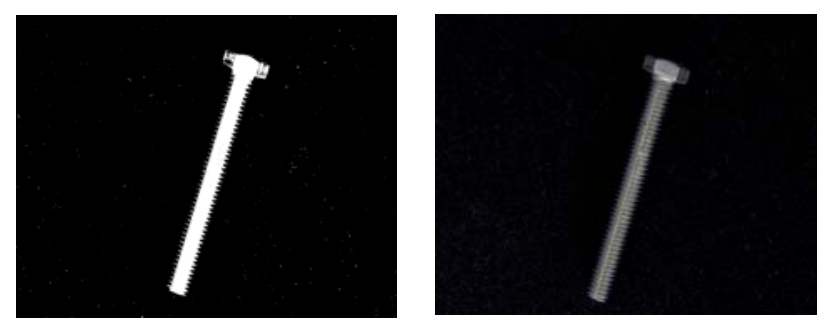

FIGURE I. GRAY-SCALE IMAGE AND BINARY IMAGE OF BOLT UNDER NATURAL LIGHT

Since the surface of the bolt reflect the light specularly, in order to separate the object to the background, black with low reflectivity is first selected as the background, and it is expected to make the brightness of the bolt higher than the background, so that the object to be tested can be separated in the step of threshold segmentation. However, when the background is black, it can be seen that the natural illumination can not make the reflectance of the bolt reach the degree of a clearly displayed edge after treatment for the light is dark, the reflection of the bolt to the light is weaker than expected, and its performance in the image is much lower then the brightness that can be clearly recognized, so it is necessary to add an additional light source on the basis of natural illumination, so that the bolt picture can be completely recognized after a series of processing such as gray leveling.

In the RGB model, when there is a $\mathrm{r}=\mathrm{g}=\mathrm{b}$ in a picture, that is, when the primary color valuesof the three constituent images are the same, the picture will no longer contain color information, but only the gray representation related to brightness. This picture is called a grayscale image ${ }^{[8]}$. Therefore, in the process of trying to recognize whether different colors of light will influence the result of the recognition, after taking the grayscale image under the illumination of different colors only, there will be seen that the difference of the bolt images under different illuminations of light is only due to the intensity. The difference is not affected by its color (Fig. 2).
(A) RED LIGHT

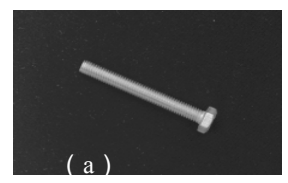

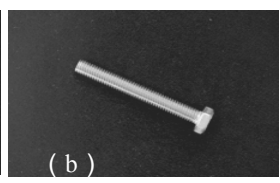

(B) BLUE LIGHT

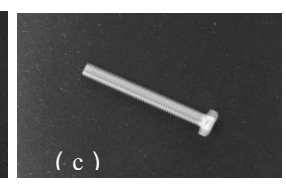

(C) GREEN LIGHT
FIGURE II. GRAY-SCALE IMAGE OF BOLTS UNDER DIFFERENT COLOR ILLUMINATION

The angle of illumination is also one of the factors that affect the final effect of the image ${ }^{[9]}$. The light will produce a shadow, and the shadow of the light will make the gray value around the bolt much smaller than the bolt itself, which will produce enough gray difference, but due to the different placement position, the bolt shadow can not be set accurately on the right place. So it will be determined by many experiments to determine which kind of illumination is suitable for the defect detection of the test object.
The single-side light source will give a shadow on one side of the bolt. The specular reflection on the surface of the bolt can make the brightness of the backlight side meet the requirement of gray level difference with the background. By using the difference between the gray level of the shadow and the bolt, a clear one-sided side of bolt profile can be obtained(Fig. 3), but the illumination on one side will make the overall gray scale of the bolt uneven. Although the two sides of the bolt have sufficient gray scale difference with the adjacent background, the overall gray scale of the bolt is not suitable. A one-sided bolt profile cannot form a closed curve in the step of contour recognition, bringing about a uncomplete bolt profile. Therefore, the requirements for illumination during the detection of bolt defects should consider minimizing the effects of single-sided shadows on the image.

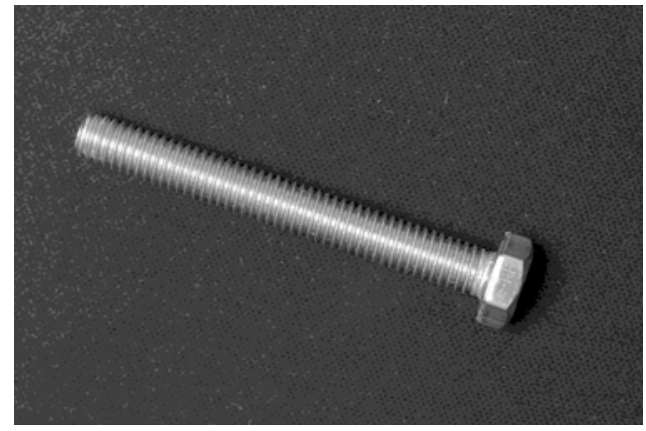

FIGURE III. GRAYSCALE DIAGRAM OF THE BOLT UNDER THE SINGLE-SIDED LIGHT SOURCE

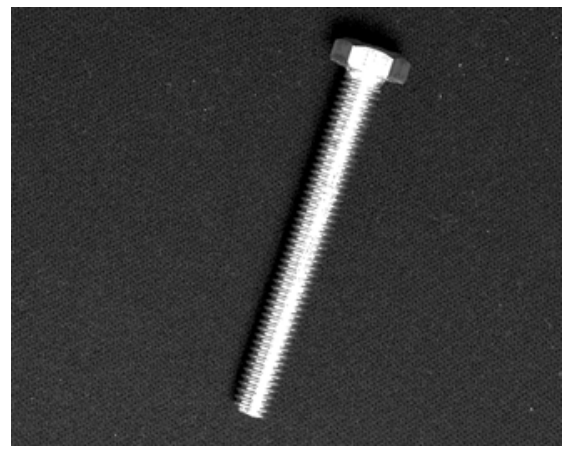

FIGURE IV. GRAYSCALE DIAGRAM OF BOLTS UNDER VERTICAL ILLUMINATION

The vertical illumination directly above can leave a complete and uniform shadow on the edge of the bolt (Fig. 4). The complete shadow ensures that the outer contour of the bolt is clearly contrasted with its adjacent shadow, but the shadow outside the bolt contour cannot make the image clearly present the contour in the image recognition alone. The uneven of the background color will cause the system to judge the wrong boundary contour in the threshold-divided, and the shadow edge is mistakenly extracted as the bolt outline. Therefore, in addition to avoiding unilateral shadows, the illumination of bolt defect detection should also avoid the occurrence of shadows that can affect contour recognition, provided that the illumination intensity is appropriate. 
The dome light source can reduce the shadow to a large extent, and the dome light source utilizes the diffuse reflection of the light, so that the light is reflected irregularly on the dome, leading the light to the object not parallelly, and the diffuse light source avoids the problems of shadows, etc. of the parallel light source. However, because of the small application range and the demanding conditions of the dome light source, when the number of objects to be tested increases, shadows will be generated between the objects to be tested, so shadows are not avoided as much as possible. There will still be a certain influence in the image, which does not meet the needs of the subject, that is able to providing conditions for satisfying the image of defect detection under the premise of the cluster (Fig. 5).Therefore, dome light source only is not suitable for the illumination of bolt defects detection in this subject.

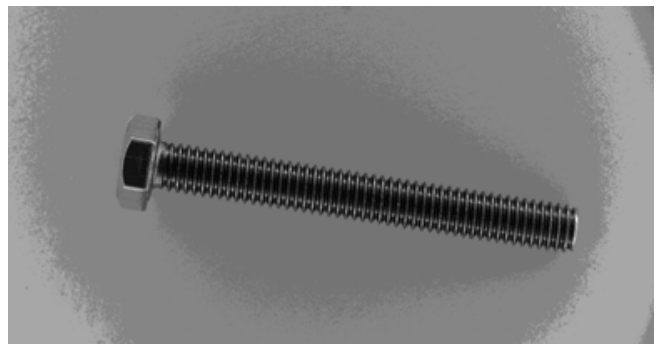

FIGURE V. GRAYSCALE DIAGRAM OF THE BOLT ILLUMINATED BY THE DOME LIGHT SOURCE

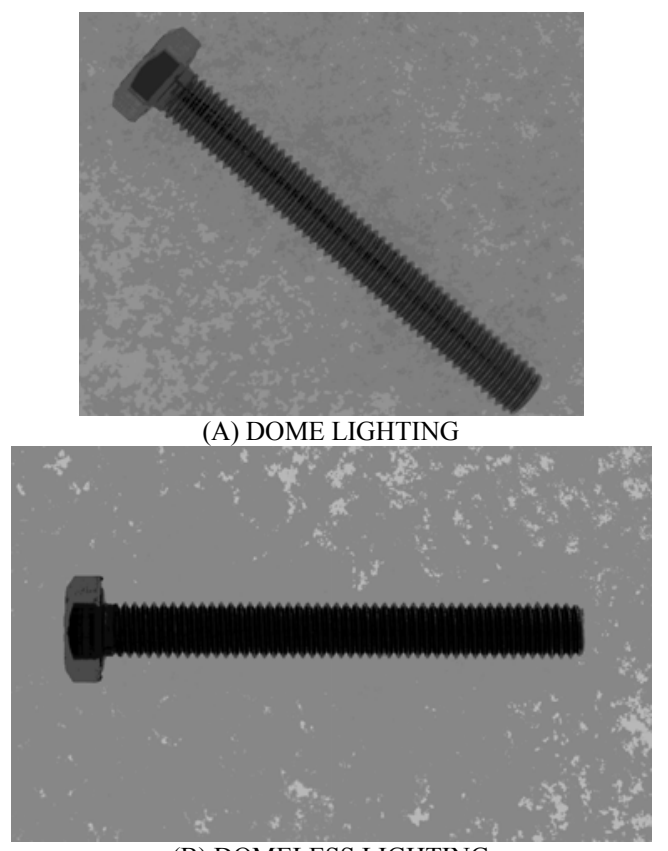

(B) DOMELESS LIGHTING

FIGURE VI. GRAY-SCALE IMAGE OF BOLTS IN BACKLIGHT

Backlighting ensures that there will be no shadows around the bolts. Therefore, backlighting is added to the dome light source during the drawing process to make sure that there is no shadow interference around the bolts, but the light source emitted from the bottom of the bolt is reflected by the dome and irradiate on the surface of the bolt ,which increases the brightness of the surface of the bolt, making the gray level of the bolts in the picture close to the one of the background, which means a reduction of the difference of gray level between the two. Therefore, it can be seen that this kind of illumination will make the bolt brightness too high, which will cause the gray value of the bolt to become larger (Fig. 6-a), and the contrast with the bolts will still be affect due to the increase of the background gray value and leading to a result of a small difference between the bolts and the background, so only backlighting is kept (Fig. 6-b).

As for the selection of background, considering that natural light was used in the initial lighting, in order to make the grayscale of the objects to be tested significantly different from the background, the background with low reflectance was selected to ensure that the background was lower than the brightness of the objects to be tested under the condition of low illumination brightness. The black background is less reflective of light and can be rendered in a smaller grayscale on the image, which is enough to differ from the specular reflection of the bolt. However, the black background cannot reflect the light uniformly under the illumination, and will generate a certain degree of reflection due to different irradiation angles. As a result, a lot of noise interference will occur after the background image is transformed into grayscale image, which will interfere with the subsequent work. Therefore, in order to completely distinguish the background from the part to be tested in the subsequent threshold segmentation step, the white background that can generate diffuse reflection is selected.

\section{IMAGE PREPROCESSING}

The grayscale image is composed of 256 grayscale values in the range of $0 \sim 255^{[10]}$, which serves as the basis for supporting image processing defect detection. The detection of defects is done by means of grayscale images. As a kind of image containing only image brightness data, the grayscale image does not contain color information, and the feature of not containing color information compresses the calculation amount, so that unnecessary influence is excluded, only the information necessary for the calculation is left. In the aspect of defect detection, obtaining the grayscale image is the first step of the calculation process.

The grayscale image can be obtained by eliminating the color information of the image. However, after image grayscale, it can be obviously found that there are visible noises in the image and insufficient grayscale difference between the object to be measured and the background, which will affect the subsequent threshold segmentation or contour extraction. Therefore, in order to deal with these cases, first of all, the obtained grayscale image will be preliminarily processed.

A common processing method in image processing is to use a specific template to operate pixels in an image. Some pixels in the gray image have an operational relationship with the adjacent or close ones. These pixels are manipulated according to their statistical properties and templates, this is called template operations or template operations ${ }^{[11]}$.

The template operation is usually used to filter the image to reduce the influence of various noises on the image. The 
common noise includes gaussian noise, uniform noise and impulse noise. Different noise will cause different results of image pollution, in the processing mode also need to use different kinds of filtering methods. No certain filtering method is suitable for noise in any case, and the selection of inappropriate filtering method will sometimes damage the picture, so the selection of filtering method and template should be decided according to its specific situation, and cannot be generalized. Mean filtering can be used when dealing with impulse noise that has little effect on the image.

Gray histogram is a table that directly reflects the gray distribution in the picture. The gray distribution of the whole picture can be judged from the gray histogram, it can visually display the pixels located in each gray level, and arrange them according to the increasing order of gray scale, and finally get the complete gray distribution histogram ${ }^{[12]}$.

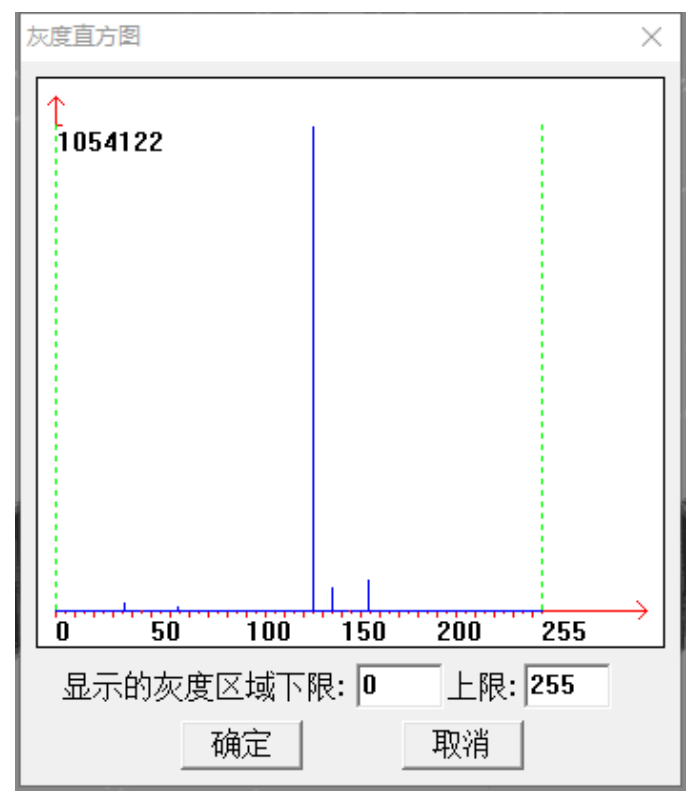

FIGURE VII. GRAY HISTOGRAM

It can be seen from Fig. 7 that the gray scale of the image is mainly concentrated in the vicinity of two gray values, which are the foreground and background of the image. As the gray difference between foreground and background is small, it is necessary to conduct gray stretching according to the distribution of gray, using the function:

$$
f(x)= \begin{cases}\frac{y_{1}}{x_{1}} x & x<x_{1} \\ \frac{y_{2}-y_{1}}{x_{2}-x_{1}}\left(x-x_{1}\right)+y_{1} & x_{1} \leq x \leq x_{2} \\ \frac{255-y_{2}}{255-x_{2}}\left(x-x_{2}\right) & x>x_{2}\end{cases}
$$

The gray scale in a certain range is uniformly enlarged to a range of 0 to 255 , Gray scale stretching is similar to the linear transformation of gray scale, both of which use gray value to carry out linear transformation, but different from gray scale linear transformation, gray scale stretching only carries out linear transformation for a part of the whole gray scale, and stretches it to the required range. $(\mathrm{x} 1, \mathrm{y} 1)(\mathrm{x} 2, \mathrm{y} 2)$ in the expression is the two endpoint coordinates of the stretched segment. This processing method can highlight the image features. In this paper, since the lighting cannot enlarge the gray difference between the object to be measured and the background, and the gray images outside the selected range will not affect the experimental results, the gray values within the designated range can be scaled up to the whole gray range by using gray scale stretching. That is to say, gray scale stretching can indeed increase the gray scale difference between the object to be measured and the background without destroying the outside contour of the object to be measured. So as to highlight the features needed for image recognition, the image can be more easily and accurately identified in the threshold segmentation of the line we need. The discontinuity of object edge grayscale produces the contour of the object. The change rate of grayscale between pixels at the edge of the object is quantified, and the detection operator is applied to calculate the contour of the object.

\section{CONTOUR ReCOGNITION AlgORITHM}

This project select the most complete and clear one as the operator used in the experiment through various operators.

The Robert operator is a $2 \times 2$ local difference operator that depends on images. In this way, the edge recognition operator simulates the process of human visual system to see objects. By using the square root operation in equation 2, Robert operator can well identify and detect the edge of images, where $f(x, y)$ is the input image with integer pixel coordinates.

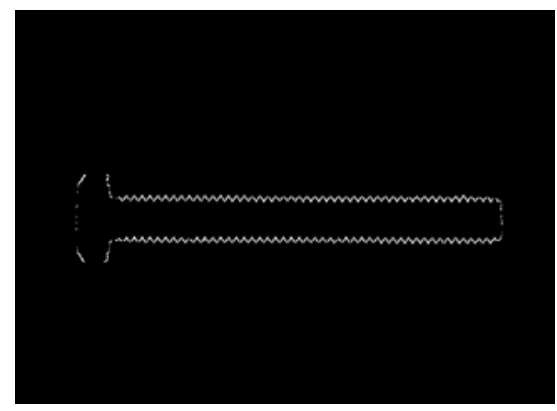

FIGURE VIII. CONTOUR RECOGNITION OF ROBERT OPERATOR

The sobel edge operator is composed of two convolution kernels, every pixel is convolved according to the two kernel, wherein one kernel responds to the vertical edges of the image, and the other kernel is for the horizontal edge of the image. The maximum value of the two convolutions is calculated as the operation result of pixel, and finally output an image with edge amplitude. 


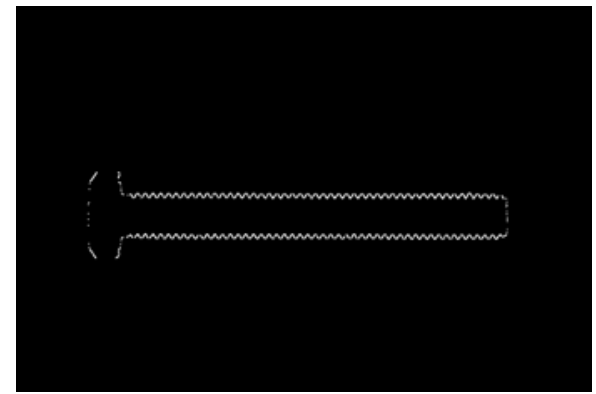

FIGURE IX. CONTOUR RECOGNITION OF SOBEL OPERATOR

The Prewirr operator is similar to the sobel operator. It is an algorithm with two convolution kernels. However, the convolution kernel it uses is different from sobel operator, and the same as sobel operator, it also calculates the maximum value of two convolution as the operation result of this point, and outputs an image of edge amplitude.

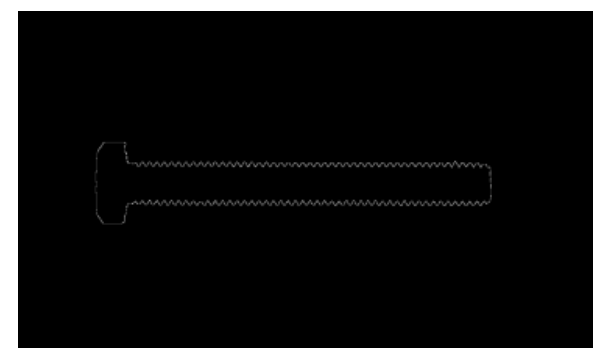

FIGURE X. CONTOUR RECOGNITION OF PERWIRR OPERATOR

The Krisch operator increased edge direction of convolution response to eight on the basis of response to the vertical and horizontal, each point in the image is used by eight mask for convolution, the eight mask as shown, each mask is corresponding to a specific direction, for this particular direction on the edge of the response of the largest, the highest value in the eight directions as the pixel output, the maximum response of mask serial number coding form the edge direction.

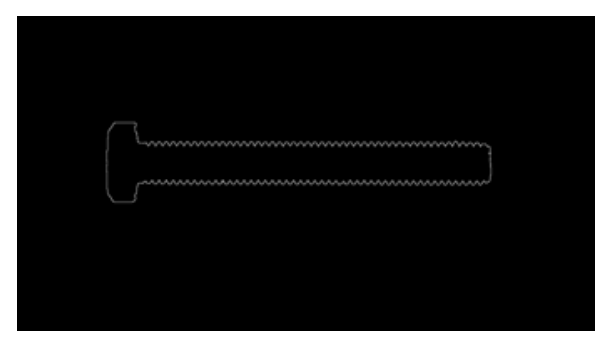

FIGURE XI. F CONTOUR RECOGNITION OF KRISCH OPERATOR

As a combination of the second derivative operator of gaussian smoothing filter and Laplace sharpening filter, Gauss - Laplacian operator uses its operations of two-dimensional function creates a steep zero crossing in the edge, for noise has a significant influence on edge detection, the operator algorithm firstly smoothing noise, then goes on the edge detection. This makes the gaussian Laplacian operator superior to other operators in processing images polluted by noise.

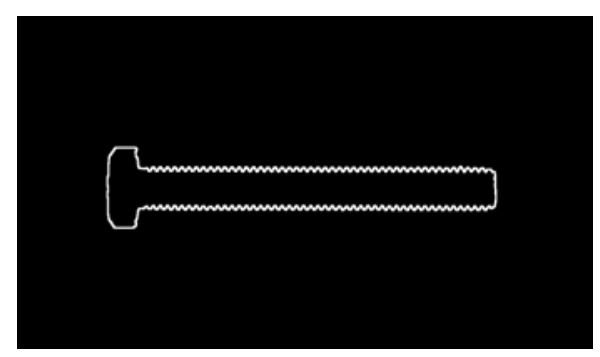

FIGURE XII. CONTOUR RECOGNITION OF GAUSS-LAPLACE OPERATOR

The Canny operator is a detection operator whose purpose is to find the optimal edge. It can detect strong edge and weak edge simultaneously by using two different threshold methods. And only when the weak edge in the image is adjacent to the strong edge will the weak edge be added to the output, so this algorithm can reduce the interference caused by noise to some extent, and can not miss the weak edge in the image in the calculation.

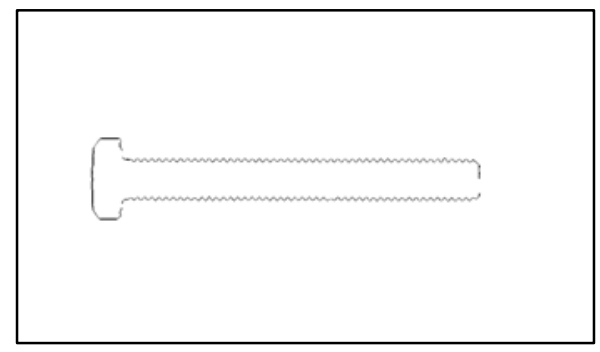

FIGURE XIII. CONTOUR RECOGNITION OF CANNY OPERATOR

\section{Defect Detection}

\section{A. Differential Method}

Defect detection based on image recognition mostly adopts difference shadow method to determine the defect. The difference shadow method uses the algebraic operation of images to subtract between the corresponding pixels of two images, and finally obtains the difference image to judge the difference between two images.

In the detection of defects, the outer contour of the defective product is different from the qualified product, it cannot be completely overlapped.so after performing the difference image operation on the two products, there will be a significant aberration image left, the number of pixels based on difference image residue can judge whether the object under test is the same as the NCM, namely whether the object under test for qualified products ${ }^{[13]}$.

\section{B. Positioning of Bolt Outlines}

The premise of the difference image operation is that the corresponding pixel points in the two subtraction images must be located at the same target point in the space. Therefore, before performing the difference image calculation, the object and the template should be positioned to match at first. Then perform the difference calculation.

Shallow threaded and unthreaded bolts can not be used as location feature points because the tooth roots are not aligned 
with the qualified parts. The intersection of the central axis of the bolt and the outer contour of the bolt is two points that are relatively fixed in the image, so it can be used to determine the position of the bolt simply and accurately.

The cluster image of the object to be measured cannot guarantee that every object to be measured can correspond to the standard template in space. Therefore, it is necessary to match the detected contour in the picture with the template at first, distinguish each individual object to be measured, and then make the two corresponding through geometric correction and registration.

The object to be measured should be separated from the image before matching and subtraction processing. Contour tracking is one of the ways to obtain the contour features of the image $e^{[14]}$.After contour extraction, the image is transformed into a binary image, that is, only images with gray scale of 0 and 255 are included. On this basis, contour tracking is carried out for the binary image. The basic method of contour tracking is to find out the pixels on the contour of the object to be tested according to a certain "probe criterion" at first, and then take corresponding "tracking criteria" according to the characteristics of the pixels to find other pixels. According to the order from left to right and bottom to top, you can find the bottom left point of the image, then take this pixel as the starting point, take the upper left as the initial search direction, search for the black pixels adjacent to the starting point in clockwise order and record their coordinates. When the entire search process returns to the original starting point, the coordinate set of the outer contour of the entire object to be tested is obtained ${ }^{[15]}$.

Hough transform is a common way to detect the straight line in the image. Taking the bolt as an example, it maps the points on the outer contour of the bolt to the Hough space with $(\gamma, \theta)$ as the parameter to obtain sinusoids. The intersection of these sinusoidal curves is a continuous or discontinuous straight line existing on the outer contour of the bolt. By narrowing the detection range to a parallel straight line, it is possible to obtain two straight lines with the axis of the bolt as the axis of symmetry between the root and the tooth of the bolt.

There are a limited number of points on the central axis of the bolt, which are output into a set. The intersection of this set and the point on the outer contour of the bolt is the intersection point of the central axis of the bolt and the outer contour of the bolt. According to the coordinates of the two intersection can determine the location of the bolt, and the slope of the line represented by this set is the rotation Angle of the picture when performing the geometric transformation, pictures will be carried out in accordance with the Angle obtained by transformation and translation, make the two points on the image to be tested coincide with the two points on the template, and then make the corresponding pixels between the two pictures coincide, so as to facilitate the realization of difference shadow calculation.

However, since there is no distinguishing feature between these two points, the correct orientation of bolts cannot be guaranteed during the geometric transformation of the picture, so the difference shadow should be calculated for several times.
The wrong orientation bolt will make the template and the bolt head in the image unable to overlap, thus leaving a large number of pixels with a gray scale of 255 . If a single result is used to judge whether the object to be tested is a defective product, misjudgment will occur. Only when the two difference shadow results are larger than the error results can we determine that the object to be measured is defective.

Figure 14-a shows the result of difference shadow after image geometric transformation when the bolt orientation of qualified product is correct. Fig. 14-b shows the result of the difference shadow after geometric transformation when the bolt orientation of qualified product is wrong. It can be clearly seen from the figure that there are a large number of white pixels on one end of the bolt when the bolt orientation is wrong, while when the bolt orientation is right, the bolt head can basically coincide, so only a small part of the error can be left due to the process and other factors.
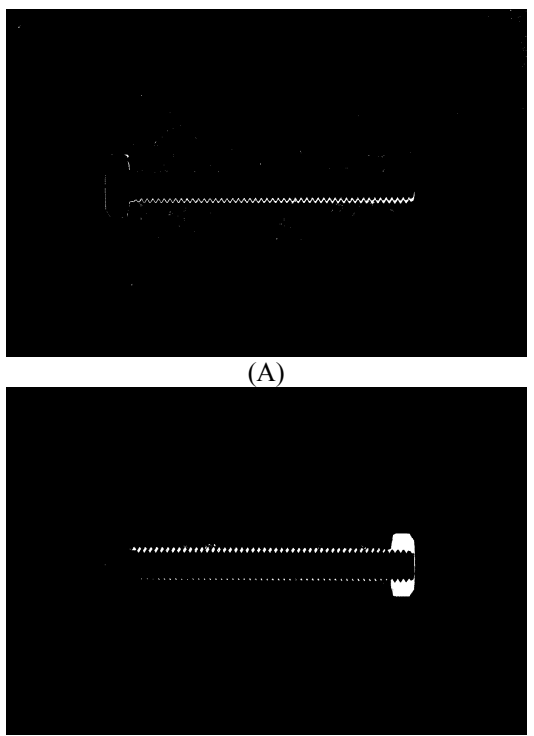

(B)

FIGURE XIV. (A) THE RESULT OF CORRECT MOVEOUT OF QUALIFIED BOLTS (B) THE RESULT OF ERROR MOVEOUT OF QUALIFIED BOLTS

Figure 15 shows the result of the shadow of the unthreaded bolt. It can be seen that when the bolt is facing the wrong direction, the bolt has a large number of white pixels as the qualified product. When the bolt is oriented correctly (FIG. 15a), the image of the bolt will not coincide with the template at the screw position, leaving two distinct white pixel bars at the edge. The two white pixel bars contain enough pixels to determine the result.

Figure 16 shows the difference between the template and the measured image under the condition of shallow thread. The determination condition is similar to that without thread, there are a large number of white pixels in the bolt head while wrong orientation, while there are still enough pixels around the stud for judgment when the orientation is correct. 


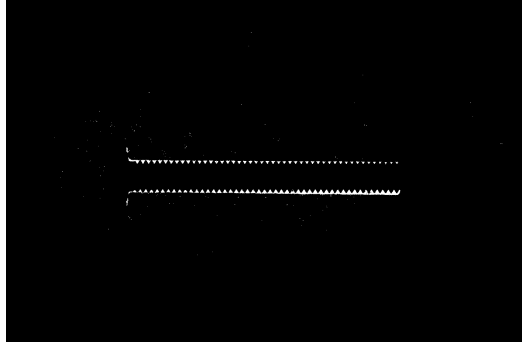

(A)

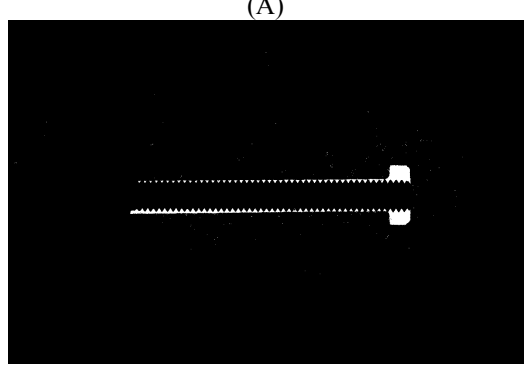

(B)

FIGURE XV. FIGURE XV(A) THE RESULT OF CORRECT MOVEOUT OF UNTHREADED BOLT (B)THE RESULT OF ERROR MOVEOUT OF UNTHREADED BOLT

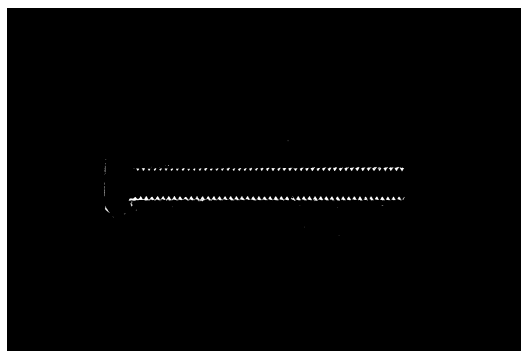

(A)

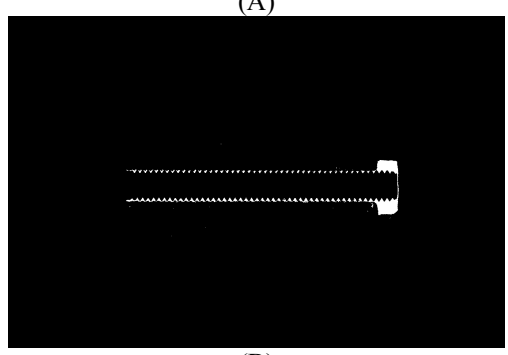

(B)

FIGURE XVI. (A)THE RESULT OF CORRECT MOVEOUT OF SHALLOW THREADED BOLT (B)THE RESULT OF ERROR MOVEOUT OF SHALLOW THREADED BOLT

\section{CONCLUSION}

Machine vision is often used in the defect detection of small parts, and backlight lighting is the most able to leave a clear outline of the lighting method, this paper compares the effects of various lighting methods in the detection of small probability parts defects, and finally decided to use backlight lighting as the experimental light source. After obtaining the image, a series of operations such as graying, noise reduction and filtering are carried out on the measured image and template image to ensure that the image can achieve the definition of contour recognition. This paper experiments the effect of a variety of operators in the defect detection of small parts. Because the defect detection of small parts requires higher accuracy, the outer contour of the image obtained by the operator used for detection must be a single pixel line, so the operator finally decided to use is canny operator. The difference between the defective part and the template is obtained by difference shadow method. The image of the object to be measured is matched with the template by taking the symmetry axis of the bolt and the straight line of the top of the screw as the characteristics, and the difference shadow calculation is made between the two. Finally, the conclusion of whether the object to be measured has defects or not is drawn based on the result of difference shadow calculation.

\section{REFERENCES}

[1] Xuanshuo, Wang Junzhen Wang Jianbin Zhang. "Ultrasonic guided wave pipeline defect detection using wavelet threshold selection method analysis" Foreign Electronic Measurement Technology 11.8 (2010):33$35+40$.

[2] Zhengbing, Shen Yuming Yang. "Techniques of machine vision applied in detection of copper strip surface's defects" Electronic Measurement Technology 33.4 (2010):65-67.

[3] LIU Gang;HUANG Yi-ming;LU Yu-ting;REN Xing-long. "Application of Machine Vision in Nut Orientation" Mechanical \& Electrical Engineering Technology 47.3 (2018):95-97..

[4] Liu, Y., J. Yang, and C. Wang. "Apple defect detection algorithm based on Convolution Neural Network." electronic Measurement Technology 40.3 (2017):108-112.

[5] ZHU, Qi-gao, and Xian-ming ZHANG. "Technology development of domestic fastener industry" Metal Products 36.1 (2010):11-13.

[6] Hu Jiacheng;Lu Feng;Chen Aijun;Chang Xu;Jin Yunchao "ABS gear ring round surface defect testing method" Journal of Electronic Measurement and Instrumentation 31.3 (2017): 408-414

[7] Xinghua, Qu, He Ying, and Han Feng. "Illumination System for Detecting Random Defects on Strongly Reflective and Complex Surfaces" Acta Optica Sinica 23.5 (2003): 547-551.

[8] Kuo-Hua, Xiao , and L. Yu . "Research of Button Cell Surface Defects Detection Algorithm" Surface Technology 42.1(2013):127-130.

[9] Tian, G. , Gao, B. , Gao, Y. , Wang, P. , \& Shi, Y. . "Review of railway rail defect non-destructive testing and monitoring" Chinese Journal of Scientific Instrument 37.8 (2016):1763-1780.

[10] Ze, Liu . "Design of machine vision system for inspection of rail surface defects" Journal of Electronic Measurement and Instrument 24.11(2010):1012-1017

[11] Cai-Xia, Z. , Gang-Yao, K. , Hai-Na, S. , \& Jiang-Yi, Y. I. . "A Fast Human Face Detection Method Based on Difference Image and Multitemplates Matching" Application Research of Computers 21.5 (2004):197-199.

[12] Tang, B. , Kong, J. Y. , Wang, X. D. , \& Chen, L. . "Surface Inspection System of Steel Strip Based on Machine Vision" 2009 First International Workshop on Database Technology and Applications IEEE, 2009.

[13] He, Z. , Wang, Y. , Liu, J. , \& Yin, F. . "Background differencing-based high-speed rail surface defect image segmentation" Chinese Journal of Scientific Instrument 37.3(2016):640-649.

[14] Zhou X. , Wang Y., Zhu Q. , Wu C. Peng Y. . "Research on defect detection method for bottle mouth based on machine vision" Journal of Electronic Measurement and Instrumentation 30.5(2016):702-713

[15] Jianping, X. , Center, I. T. , \& Polytechnic, S. . "Research on the surface defect detection of floor tile based on computer vision" 38.5(2015):5355. 